\title{
EFFECTIVE GROWTH RATE OF WHITE DWARF MASS IN NOVA OUTBURSTS
}

\author{
Mariko KATO \\ Department of Astronomy, Keio University \\ Hiyoshi, Kouhoku-ku, Yokohama 223, Japan \\ and \\ Izumi HACHISU \\ Department of Aeronautical Engineering, Kyoto University, Kyoto 606, Japan
}

\begin{abstract}
We have obtained the effective growth rate of white dwarf masses which are suffering mass loss during both hydrogen and helium nova outbursts. If the mass transfer rate from the companion is smaller than $10^{-7} \mathrm{M}_{\odot} / \mathrm{yr}$, the net growth rate is reduced to less than one tenth of the mass transfer rate from the companion star. It is suggested that the white dwarf mass is hard to grow to the Chandrasekhar mass unless its initial mass is very close to the Chandrasekhar limit.
\end{abstract}

It has been argued that a nova/recurrent nova system is a progenitor of Type Ia supernova. If the white dwarf, which is accreting matter from the companion, can grow to the Chandrasekhar mass, a Type Ia supernova explosion is expected. Along the same type of scenario, it has been also suggested that neutron stars in low-mass binaries are formed through the accretion-induced collapse of white dwarfs. Both scenarios are essentially based on the assumption that the white dwarf mass can grow to the Chandrasekhar mass. It is, however, not yet justified because the large amount of the hydrogen-rich envelope or the helium envelope of the white dwarf will certainly be lost from the system during the nova outburst.

We have considered two mechanisms of mass-stripping: one is the optically thick wind mass loss and the other is the Roche lobe overflow. The optically thick wind can be followed by Kato's steady state approach (Kato 1983; Kato and Hachisu 1988, 1989; Kato, Saio, and Hachisu 1989). In this method, mass loss rates can be accurately determined as an eigenvalue of equations. Using these mass loss rate, we have obtained the net growth rate of the white dwarf mass. The net accretion is characterized by the mass accumulation ratio $\eta$, that is, the ratio of the processed matter accreted by the white dwarf to the mass transferred from the companion star. The mass accumulation ratio is plotted in Figure 1 against the mass transfer rate from the companion for both hydrogen and helium burning outbursts (labeled by $\mathrm{H}_{\infty}$ and $\mathrm{He}_{\infty}$, respectively). Helium shell flashes are expected to occur after many hydrogen shell flashes (i.e., after many nova cycles). The wind mass loss during helium shell flashes is important around the mass transfer rates $10^{-8} M_{\odot} / \mathrm{yr}<\dot{M}<10^{-7} M_{\odot} / \mathrm{yr}$. Only a very massive white dwarf with a high mass transfer rate is possible to grow to the Chandrasekhar mass if the companion is a low-mass star. These systems are probably recurrent nova systems. Therefore, one must take into account the wind mass loss during helium shell flashes to examine whether the recurrent nova systems can explode as a Type Ia supernova or not (e.g., Hachisu, Kato, and Saio 1989). 


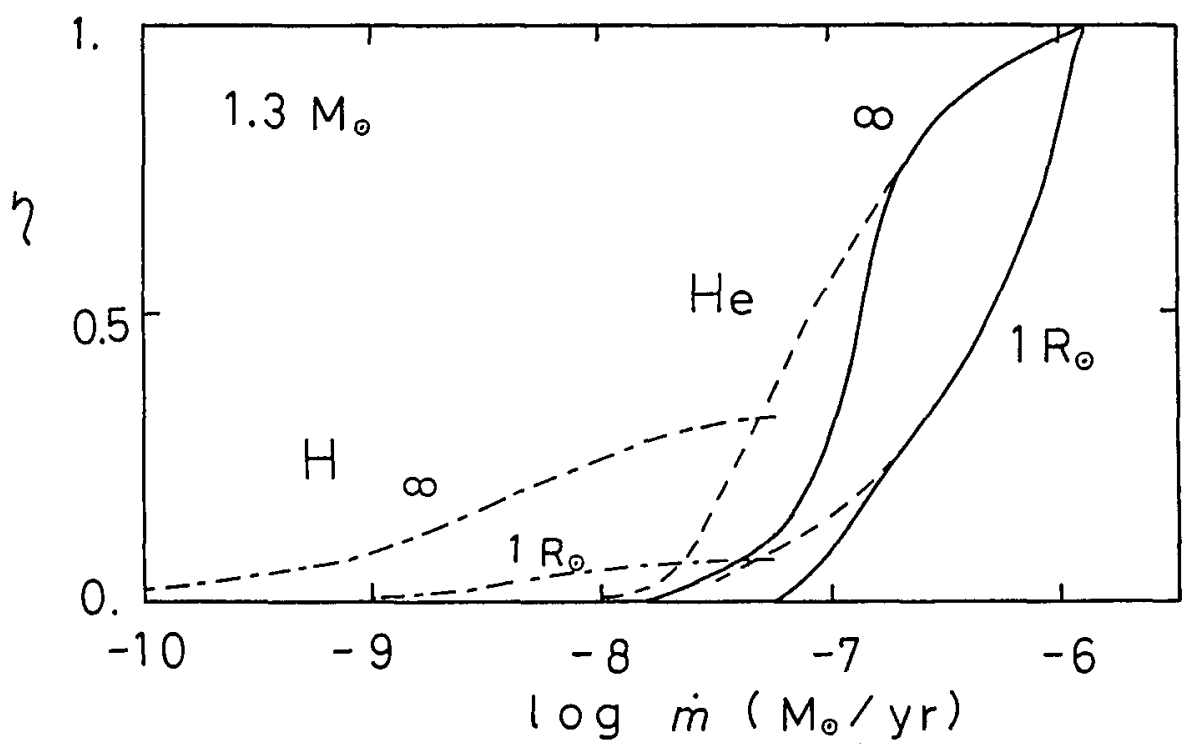

Fig. 1-Total mass accumulation ratio $\eta$ is plotted against the mass transfer rate from the companion $\dot{m}$ for a $1.3 M_{\odot}$ white dwarf. The mass accumulation ratios for hydrogen (dash-dotted, labeled by $\mathrm{H}$ ) and for helium (dashed, labeled by $\mathrm{He}$ ) are also added. Symbols attached to each line denote the size of the Roche lobe $\left(\infty\right.$ and $\left.1 R_{\odot}\right)$.

When the Roche lobe size is small enough (say, $<10 R_{\odot}$ ), the mass-stripping due to the Roche lobe overflow dominates the wind mass loss. As a result, the mass accumulation ratio for such a case is much reduced as shown in Figure 1 (labeled by $1 \mathrm{R}_{\odot}$ ).

\section{REFERENCES}

Hachisu, I., Kato, M., and Saio, H. 1989, Ap. J., 342, L19.

Kato, M. 1983, Pub. Astr. Soc. Japan, 35, 507.

Kato, M., and Hachisu, I. 1988, Ap. J., 329, 808.

Kato, M., and Hachisu, I. 1989, Ap. J., 346, in press.

Kato, M., Saio, H., and Hachisu, I. 1989, Ap. J., 340, 509. 